\title{
Hopping dynamics of interacting polarons
}

\author{
S. Ciuchi ${ }^{1,2}$ and S. Fratini ${ }^{3,4}$ \\ ${ }^{1}$ Dipartimento di Fisica and CNISM, Università dell'Aquila, via Vetoio, I-67010 L'Aquila, Italy \\ ${ }^{2}$ SMC Research Center, INFM-CNR, Piazzale A. Moro I-00185 Rome, Italy \\ ${ }^{3}$ Institut Néel, CNRS \& Université Joseph Fourier, BP 166, F-38042 Grenoble Cedex 9, France \\ ${ }^{4}$ Instituto de Ciencia de Materiales de Madrid, CSIC, Sor Juana Inés de la Cruz 3, E-28049 Madrid, Spain
}

(Received 2 October 2008; revised manuscript received 10 November 2008; published 16 January 2009)

\begin{abstract}
We derive an effective cluster model to address the transport properties of mutually interacting small polarons. We propose a decoupling scheme where the hopping dynamics of any given particle is determined by separating out explicitly the degrees of freedom of its environment, which are treated as a statistical bath. The general cavity method developed here shows that the long-range Coulomb repulsion between the carriers leads to a net increase in the thermal activation barrier for electrical transport and hence to a sizable reduction in the carrier mobility. A mean-field calculation of this effect is provided based on the known correlation functions of the interacting liquid in two and three dimensions. The present theory gives a natural explanation of recent experiments performed in organic field-effect transistors with highly polarizable gate dielectrics and might well find application in other classes of polaronic systems such as doped transition-metal oxides.
\end{abstract}

DOI: 10.1103/PhysRevB.79.035113 PACS number(s): 71.10. - w, 71.38.Ht, 73.40. -c, 72.80.Le

\section{INTRODUCTION}

In recent years, the development of organic electronics has triggered a strong effort toward the understanding of charge transport in organic field-effect transistors (OFETs). ${ }^{1}$ In such devices, the carriers induced by a gate potential move at the interface between an organic semiconductor and a dielectric. Unlike their inorganic counterparts, such as $\mathrm{Si}$ metal-oxide-semiconductor field-effect transistors (MOSFETs), the transport properties in OFETs are dominated by the weak transfer integrals between the molecular constituents of the organic material: the van der Waals intermolecular bonding leads even in pure crystalline samples to extremely narrow electronic bands, making such systems very sensitive to interactions.

Recently, a systematic study of rubrene-based singlecrystalline OFETs fabricated using gate materials of increasing dielectric polarizability has revealed that in the case of high- $\kappa$ dielectrics, the dominant limiting mechanism of electron transport originates from the coupling with the polar phonons at the organic-dielectric interface. ${ }^{2}$ This phenomenon, which in wide-band inorganic semiconductors ${ }^{3-7}$ and in graphene ${ }^{8}$ only leads to minor modifications of the electron mobility, can be so effective in organic semiconductors that it leads to polaronic self-localization of the carriers on the scale of one or few molecules. As a consequence, the mobility is strongly suppressed and becomes thermally activated, being due to the incoherent hopping of small polarons on the molecular lattice.

Because of the increased capacitance of the devices, the use of high- $\kappa$ dielectrics also has a second interesting consequence, as it allows the injection of sufficiently large charge densities, such that the electrons can no longer be considered as noninteracting carriers. ${ }^{9}$ Indeed, concentrations on the order of 0.1 carriers/molecule and above have been reached in rubrene devices using $\mathrm{Ta}_{2} \mathrm{O}_{5}$ as a gate material (dielectric constant $\epsilon_{s}=25$ ). The current-voltage characteristics of such devices exhibit strong deviations from linearity that cannot be explained in terms of independent carriers and have been ascribed to the onset of electron-electron (e-e) interactions. ${ }^{9}$

The aim of this work is to establish a theory for the density-dependent transport properties of mutually interacting small polarons in the hopping regime. Although the present derivation is motivated by the physics of organicdielectric interfaces, the problem itself is sufficiently general to find application in other polaronic systems such as transition-metal oxides $^{10}$ and possibly oxide-oxide interfaces $^{11}$ and organic charge-transfer interfaces. ${ }^{12}$ As it will become clear in the following, however, OFETs are ideal systems for the observation of the many-body effects studied here for two reasons. First, the effect of interactions on the polaronic hopping rates can be comparatively large in organic semiconductors, where small polarons can exist with activation energies that are generally smaller than in oxides. Second, and most importantly, in such devices the carrier concentration can be varied accurately by tuning the gate voltage without the need of chemical substitution, ${ }^{1,13}$ thus providing a reliable and unambiguous procedure to disentangle many-body effects from the properties of individual polarons.

The present paper provides a detailed derivation of the theory that was used in Ref. 9 to explain the current-voltage characteristics of rubrene-based high- $\kappa$ OFETs, as well as its generalization to three-dimensional (3D) systems. We start by introducing an effective cluster model that allows to separate the dynamics of any given electron from the remaining carriers in the system, which are treated as an external statistical environment. The mobility is then obtained from a suitable statistical average of the intermolecular hopping rates, assuming a succession of incoherent hopping events. The theory is applied to the calculation of the densitydependent transport properties of small polarons interacting through long-range Coulomb potentials. It is shown that the mutual interactions between carriers give rise to a net increase in the polaronic thermal activation barrier and consequently to a sizable reduction in the mobility. A mean-field calculation is performed, providing a closed-form analytical 
expression for the mobility based on the known pair distribution function of the interacting liquid.

The paper is organized as follows. In Sec. II we carry out explicitly the separation between the cluster degrees of freedom and those of the environment. In Sec. III we focus specifically on a two-site cluster, which is appropriate in the small-polaron limit. We derive a general formula for the hopping mobility of interacting small polarons and provide a simplified expression describing carrier-carrier correlations in the mean-field approximation. In Sec. IV the theory is applied to the problem of polarons interacting via the longrange Coulomb repulsion. The results are discussed in relation to the transport properties of OFETs and of other known polaronic systems in Sec. V.

\section{EFFECTIVE CLUSTER MODEL}

Let us consider a system of electrons moving on a lattice (defined by the lattice vectors $\mathbf{R}_{i}$ ) which interact with each other and with the lattice vibrations, as described by the following Hamiltonian:

$$
H=H_{t}+H_{\mathrm{ph}}+H_{\mathrm{int}}+H_{\mathrm{e}-\mathrm{ph}} .
$$

The first term

$$
H_{t}=-t \sum_{\langle i j\rangle} c_{i}^{\dagger} c_{j}
$$

is the tight-binding Hamiltonian for free electrons, with $t$ as the hopping integral and $c_{i}$ and $c_{i}^{\dagger}$ as the corresponding destruction and creation operators for electrons. The second term

$$
H_{\mathrm{ph}}=\sum_{j} \frac{1}{2} k X_{j}^{2}+\sum_{j} \frac{1}{2 M} P_{j}^{2}
$$

describes local (dispersionless) phonons of frequency $\omega_{0}$ $=\sqrt{k / M}$, where $X_{j}$ is the local lattice displacement, $P_{j}$ the conjugate momentum, $k$ is the spring constant, and $M$ is the mass. The term

$$
H_{\mathrm{int}}=\frac{1}{2} \sum_{i, j} n_{i} V_{i j} n_{j}
$$

is the e-e interaction where $n_{i}=c_{i}^{\dagger} c_{i}$ is the electron-density operator at site $i$, and $V_{i j}=V\left(\mathbf{R}_{i}-\mathbf{R}_{j}\right)$ is a generic densitydensity interaction potential. Finally,

$$
H_{\mathrm{e}-\mathrm{ph}}=\sum_{i, j} n_{i} g_{i j} X_{j}
$$

is the electron-phonon (e-ph) interaction where $g_{i j}=g\left(\mathbf{R}_{i}\right.$ $-\mathbf{R}_{j}$ ) is a non-local-density-displacement coupling. We shall not give here the precise form of $g_{i j}$ and $V_{i j}$, which is not needed for the general formalism developed in the following paragraphs. This will be provided later when dealing with specific examples.

\section{A. Derivation of the cluster Hamiltonian}

We now derive an effective cluster model neglecting the second term in Eq. (3), which is valid at temperatures $T$ $\gtrsim \omega_{0}$. The phonon kinetic-energy term will be reintroduced in Sec. II B to treat the polaron hopping dynamics in the semiclassical adiabatic approximation.

Our starting point to evaluate the hopping mobility of the system described by Eq. (1) is to divide the lattice into a cluster $(c)$ of finite size, in which we allow for quantum electron hopping and a remaining part $(\bar{c})$ in which the dynamics of the electrons is neglected. This separation is enforced by keeping a finite transfer integral $t$ only for electrons within the cluster $(c)$, while setting $t=0$ in $(\bar{c})$. It is then natural to rewrite model (1) by singling out the terms which explicitly contain electronic variables in $(c)$ that we denote as $H_{\text {. }}$

$$
H_{\bullet}=H_{t}^{(c)}+H_{\mathrm{int}}^{(c)}+H_{\mathrm{e}-\mathrm{ph}}^{(c)}+H_{\mathrm{int}}^{(c, \bar{c})}
$$

so that

$$
H=H_{\mathrm{ph}}+H_{\mathrm{int}}^{(\bar{c})}+H_{\mathrm{e}-\mathrm{ph}}^{(\bar{c})}+H_{. .}
$$

In Eqs. (6) and (7) the labels $(c)$ and $(\bar{c})$ indicate that the sums over electronic variables are restricted, respectively, to the cluster or the environment, and $(c, \bar{c})$ stands for interactions among electrons belonging to the two different subsystems. It can be noted that all terms in Eq. (7) commute with each other.

The proposed separation scheme is formally equivalent to the one used by Pardee and Mahan ${ }^{14,15}$ to describe electrical conduction in solid electrolytes. The justification in that case follows from the large masses of the charge carriers that are mobile ions. As a result, the collective rearrangement of the particles in reaction to a given hopping event is much slower than the hopping process itself, so that the positions of the carriers in the environment can effectively be regarded as static variables during the local dynamical evolution. In our case this decoupling is justified due to the exponential suppression of the carriers' hopping rate associated to polaronic self-localization.

To derive an effective cluster model, it is useful to introduce the following reduced density matrix:

$$
\rho_{r}=\frac{1}{Z} \operatorname{tr}_{(\mathrm{ph})} \operatorname{tr}_{(\bar{c})} e^{-H / k_{B} T} \prod^{(c)} \delta\left(Y_{i}-\sum_{j} g_{i j} X_{j}\right),
$$

where $\Pi^{(c)}$ indicates the product over the cluster electrons, the trace symbols are defined as

$$
\begin{aligned}
& \operatorname{tr}_{(\bar{c})}(\cdots)=\sum_{n_{i}, i \in(\bar{c})}(\cdots), \\
& \operatorname{tr}_{(\mathrm{ph})}(. .)=\int \prod_{i} d X_{i}(. .),
\end{aligned}
$$

and $Z=\operatorname{tr}_{(c)} \operatorname{tr}_{(\mathrm{ph})} \operatorname{tr}_{(\bar{c})} e^{-H / k_{B} T}$. In Eq. (9) we trace over all the electronic degrees of freedom which do not belong to the cluster. The trace over phononic variables in Eq. (10) is performed by assigning the value of the phonon-induced external fields, 


$$
Y_{i}=\sum_{j} g_{i j} X_{j}, \quad i \in(c) .
$$

These are the phononic collective variables, which act on each site of the cluster, through nonlocal e-ph interaction (5). The two steps described above are now explicitly carried out.

\section{Tracing out the phonons}

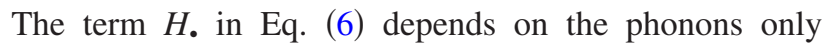
through the variables $\left\{Y_{i}\right\}$. We can therefore rewrite Eq. (8) as

$$
\begin{aligned}
\rho_{r}= & \frac{1}{Z} \operatorname{tr}_{(\bar{c})} \operatorname{tr}_{(\mathrm{ph})} e^{-\left(H \cdot\left(\left\{Y_{i}\right\}\right)+H_{\mathrm{int}}^{(\bar{c})}\right) / k_{B} T} \\
& \times \prod_{i}^{(c)} \delta\left(Y_{i}-\sum_{j} g_{i j} X_{j}\right) e^{-\left(H_{\mathrm{ph}}+H_{e-\mathrm{ph}}^{(\bar{c})}\right) / k_{B} T},
\end{aligned}
$$

where with $H_{0}\left(\left\{Y_{i}\right\}\right)$ we indicate the explicit dependence of this term on the collective phonon variables. The trace over the original phonons $X_{i}$ in Eq. (12) can be performed by introducing the integral representation of the $\delta$ function,

$$
\delta\left(Y_{i}-\sum_{j} g_{i j} X_{j}\right)=\int \frac{d \omega_{i}}{2 \pi} \exp i \omega_{i}\left(Y_{i}-\sum_{j} g_{i j} X_{j}\right) .
$$

Performing the Gaussian integrals over $\left\{X_{i}\right\}$ and over $\left\{\omega_{i}\right\}$, we obtain

$$
\rho_{r} \propto \operatorname{tr}_{(\bar{c})} e^{-\left[H_{.}(Y)+H_{\mathrm{int}}^{(\bar{c})}+H_{\mathrm{eff}}^{(\bar{c})}\right] / k_{B} T},
$$

where $H_{\text {eff }}^{\bar{c}}$ represents the effective Hamiltonian resulting from the trace over phonons. It can be expressed as

$$
H_{\mathrm{eff}}^{(\bar{c})}=-\frac{1}{2} \sum_{i, j}^{(\bar{c})} n_{i} D_{i j} n_{j}+H_{\mathrm{eff}}^{\prime(\bar{c})},
$$

with

$$
\begin{gathered}
D_{i j}=\frac{1}{k}\left[g^{2}\right]_{i j}-\frac{1}{k} \sum_{l, k}^{(c)}\left[g^{2}\right]_{i l}\left[g_{c}^{-2}\right]_{l k}\left[g^{2}\right]_{k j}, \\
H_{\mathrm{eff}}^{\prime(\bar{c})}=\frac{k}{2} \sum_{i, j}^{(c)}\left[g_{c}^{-2}\right]_{i j} Y_{i} Y_{j}+\sum_{i}^{(c)} \sum_{j}^{(\bar{c})} G_{i j} Y_{i} n_{j}, \\
G_{i j}=\sum_{l}^{(c)}\left[g_{c}^{-2}\right]_{i l}\left[g^{2}\right]_{l j} .
\end{gathered}
$$

In the above equations we have introduced the symbol $\left[g_{c}^{-2}\right]$ to denote the inverse of the matrix $\left[g^{2}\right]$ in the cluster subspace. As can be seen from Eq. (15), integrating out the phonon variables has led to an effective attraction $D_{i j}$ between the $(\bar{c})$ electrons, whose form is given by Eq. (16). Similarly, Eqs. (17) and (18) describe the effective interactions arising between the $(\bar{c})$ electrons and the collective variables $Y_{i}$.

Adding the phonon-mediated interaction of Eq. (15) to the bare electron-electron term $H_{\text {int }}^{(\bar{c})}$ in Eq. (7) yields the follow- ing screened interaction between the environment electrons:

$$
\widetilde{H}_{\mathrm{int}}^{(\bar{c})}=\frac{1}{2} \sum_{i, j} n_{i}\left(V_{i j}-D_{i j}\right) n_{j} .
$$

The reduced density matrix can be finally expressed as

$$
\rho_{r} \propto \operatorname{tr}_{(\bar{c})} e^{-\left[H_{\cdot}(Y)+\tilde{H}_{\text {int }}^{(\bar{c})}+H_{\mathrm{eff}}^{\prime(\bar{c})}\right] / k_{B} T} .
$$

Before moving on to the integration of the environment electrons, it is useful to comment on the physical meaning of the two different contributions to the phonon-induced screening in Eq. (16). The first term, which leads to the effective potential

$$
\tilde{V}_{i j}=V_{i j}-\frac{\left[g^{2}\right]_{i j}}{k},
$$

represents the ability of the polarizable medium to partially screen the electron-electron interaction. For example, starting from the bare Coulomb potential $V_{i j}=e^{2} /\left(\epsilon_{\infty} R_{i j}\right)$ and an electron-phonon interaction $g_{i j}$ of the Fröhlich type, it is shown in Appendix B that the inclusion of this term yields $\tilde{V}_{i j}=e^{2} /\left(\epsilon_{s} R_{i j}\right)$, which correctly reproduces the static screening response of a bulk polar dielectric.

The second term in Eq. (16) is a cavity field which arises due to the constraints in Eq. (11) because not all of the phonons have been integrated out. It can be viewed as the part of phonon screening that is missing due to the existence of the cluster. Since it involves the product of two matrices $\left[g^{2}\right]_{i l}\left[g^{2}\right]_{k j}$, which decays faster than the direct screening $\left[g^{2}\right]_{i j}$ itself, this term becomes negligible when the cluster size is smaller than the average interparticle distance. This cavity correction can therefore be neglected to the lowest order in the electron concentration, although its actual magnitude depends on the shape of the electron-phonon interaction $g_{i j}$ (for example, such cavity field is clearly absent in the limit of local e-ph interactions, i.e., $g_{i j} \propto \delta_{i j}$ ). On the other hand, if the cluster is enlarged to attain the size of the entire system, the two terms in Eq. (16) exactly cancel and only the bare electron-electron interaction remains.

\section{Tracing out the electronic environment}

The trace appearing in Eq. (20) can be formally carried out by introducing two classical fields which couple linearly to the cluster variables $Y_{i}$ and $n_{i}$, namely,

$$
\begin{aligned}
\eta_{i} & =\sum_{j}^{(\bar{c})} G_{i j} n_{j}, \quad i \in c, \\
\epsilon_{i} & =\sum_{j}^{(\bar{c})} V_{i j} n_{j}, \quad i \in c .
\end{aligned}
$$

Such fields take into account the interactions between electrons in $(\bar{c})$ and the cluster degrees of freedom, as contained explicitly in $H_{\mathrm{eff}}^{\prime(\bar{c})}$ and in the direct term $H_{\mathrm{int}}^{(c, \bar{c})}$. Substituting these definitions into Eqs. (6) and (17) and regrouping terms in Eq. (20), one obtains the following cluster Hamiltonian: 


$$
\begin{aligned}
H_{\text {cluster }}= & -t \sum_{\langle i j\rangle}^{(c)} c_{i}^{\dagger} c_{j}+\frac{1}{2} \sum_{i, j}^{(c)} n_{i} V_{i j} n_{j}+\sum_{i}^{(c)} n_{i} \epsilon_{i}+\sum_{i}^{(c)} Y_{i}\left(\eta_{i}+n_{i}\right) \\
& +\frac{k}{2} \sum_{i, j}^{(c)}\left[g_{c}^{-2}\right]_{i j} Y_{i} Y_{j}
\end{aligned}
$$

Finally, by enforcing definitions (22) and (23) through the appropriate $\delta$ functions, the reduced density matrix of the cluster can be expressed as a trace over the classical variables $\eta_{i}, \epsilon_{i}$ of the environment,

$$
\rho_{r}=\int \prod_{i}^{(c)} d \eta_{i} d \epsilon_{i} e^{-H_{\text {cluster }} / k_{B} T} P\left(\left\{\epsilon_{i}\right\},\left\{\eta_{i}\right\}\right),
$$

whose statistical distribution is

$$
\begin{aligned}
P\left(\left\{\epsilon_{i}\right\},\left\{\eta_{i}\right\}\right) \propto & \operatorname{tr}_{(\bar{c})} e^{-\widetilde{H}_{\mathrm{int}}^{(\bar{c})} / k_{B} T} \prod_{i}^{(c)} \delta\left(\eta_{i}-\sum_{j}^{(\bar{c})} G_{i j} n_{j}\right) \\
& \times \delta\left(\epsilon_{i}-\sum_{j}^{(\bar{c})} V_{i j} n_{j}\right) .
\end{aligned}
$$

To summarize, Eqs. (24)-(26) describe a finite cluster in which electrons mutually interact via the bare potential $V_{i j}$ and are coupled to collective phonon variables $Y_{i}$. The cluster degrees of freedom are also subject to random fields $\eta_{i}$ and $\epsilon_{i}$ arising from the environment electrons. Such fields are distributed, via Eq. (26), according to the equilibrium distribution of classical particles interacting through the screened Hamiltonian $\tilde{H}_{\text {int }}^{(\bar{c})}$ defined in Eq. (19).

\section{B. Ehrenfest dynamics of the cluster model}

Within the adiabatic regime, the carrier motion is constrained to follow the slow dynamics of the phonon coordinates. $^{10,16}$ To determine the polaron mobility it is therefore necessary to treat explicitly the dynamics of the $X_{i}$ that was neglected in Sec. II A. This can be done by introducing the semiclassical evolution of the lattice degrees of freedom through the following Ehrenfest equation:

$$
M \ddot{X}_{i}=-k X_{i}-\sum_{j} g_{i j}\left\langle n_{j}(t)\right\rangle .
$$

In the above equation the average of the electronic operators is taken at fixed $X_{i}$. To change to the cluster variables $Y_{i}$, we substitute Eq. (27) into Eq. (11) for $i \in(c)$, leading to

$$
M \ddot{Y}_{i}=-k Y_{i}-\sum_{j}^{(c)}\left[g_{c}^{2}\right]_{i j}\left\langle n_{j}(t)\right\rangle-\sum_{j}^{(\bar{c})}\left[g^{2}\right]_{i j}\left\langle n_{j}\right\rangle,
$$

where we have made explicit use of the assumption that the environment electrons do not evolve in time. Using Eq. (22) this can be rewritten as

$$
M \ddot{Y}_{i}=-k Y_{i}-\sum_{j}^{(c)}\left[g_{c}^{2}\right]_{i j}\left[\left\langle n_{j}(t)\right\rangle+\eta_{j}\right] .
$$

The collective phonon variables $Y_{i}$ are therefore subject to an external force which depends both on the instantaneous elec- tron density within the cluster and on the environment degrees of freedom through the fields $\eta_{i}$. It is interesting to observe that in the present treatment, the frequency of the collective modes is equal to the bare phonon frequency $\omega_{0}$ $=\sqrt{k / M}$. The above Eq. (29) can equivalently be derived in a Hamiltonian formulation by adding a kinetic term $(2 M)^{-1} \Sigma_{i j}^{(c)}\left[g_{c}^{2}\right]_{i j} \Pi_{i} \Pi_{j}$ to Eq. (24), with $\Pi_{i}$ as the momentum conjugate to $Y_{i}$.

\section{SMALL-POLARON LIMIT}

The actual choice of the cluster size for practical calculations is dictated by the polaron properties, since it should be large enough to accommodate the electronic wave function involved in the hopping process. To keep the discussion simple and provide a physically significant example of the theory presented so far, we now focus specifically on the small-polaron limit, where the electronic wave function collapses onto a single molecule. This situation is realized in systems with narrow electronic bands provided that the electron-phonon coupling is sufficiently strong. To be specific, this occurs when the energy of a polaron fully localized on a single molecular site $E_{P}=\left[g^{2}\right]_{11} / 2 k$ is larger than approximately half the free-electron bandwidth; in which case a self-localized state becomes energetically more favorable than an extended wave. Such condition is met at rubrene/ $\mathrm{Ta}_{2} \mathrm{O}_{5}$ interfaces, as was shown in Refs. 2 and 9, as well as in a variety of bulk transition-metal oxides. ${ }^{10}$ The proper cluster in this case consists of two molecules-the initial (filled) site and the final (empty) site-and constitutes the basis for the theory of small-polaron transport. ${ }^{10,16,17} \mathrm{We}$ shall explicitly consider situations where the formation of bipolaronic states is ruled out by the presence of sufficiently strong repulsive interactions between the carriers. ${ }^{18,19}$ Apart from this restriction, the results obtained in this section concerning the effect of electron-electron interactions will be generally valid regardless of the physical origin and particular form of $g_{i j}$; the only requirement being that the polarons are small.

\section{A. Two-site cluster}

It is shown in Appendix A that for a singly occupied two-site cluster, Hamiltonian (24) reduces to a spin-boson model, where the electronic degree of freedom plays the role of a pseudospin. Introducing the notation $\sigma_{z}=n_{1}-n_{2}$ and $\sigma_{x}$ $=c_{1}^{+} c_{2}+c_{2}^{+} c_{1}$, we obtain

$$
H_{\mathrm{sb}}=-t \sigma_{x}+\frac{1}{2} k Q^{2}-\frac{1}{\sqrt{2}}(g Q+\xi) \sigma_{z} .
$$

The relative electronic occupation $\sigma_{z}$ is coupled to the phonons through a single "interaction coordinate,"

$$
Q=\frac{Y_{2}-Y_{1}}{\sqrt{2} g}+\frac{g}{k} \frac{\eta_{2}-\eta_{1}}{\sqrt{2}} .
$$

The first term in Eq. (31) is the direct interaction with the collective phonons and the second term originates from the residual electron-phonon interaction of Eq. (18), $\eta_{1}$ and $\eta_{2}$ 
are being defined by Eq. (22). The parameter $g=\left[g^{2}\right]_{11}$ $-\left[g^{2}\right]_{12}$ is an effective electron-phonon coupling for the twosite cluster. The coupling with the environment electrons $(\bar{c})$ also occurs via a single classical variable,

$$
\xi=\left(\epsilon_{2}-\epsilon_{1}\right)-\frac{g^{2}}{k}\left(\eta_{2}-\eta_{1}\right),
$$

which takes into account the electronic repulsion $\epsilon_{i}$ on the two sites corrected by the appropriate phonon-mediated attractive terms $\eta_{i}$. Using Eq. (21), this can be rewritten as

$$
\xi=\sum_{j}^{(\bar{c})}\left[\tilde{V}_{2, j}-\tilde{V}_{1, j}\right] n_{j} .
$$

Such "local field" represents the energy unbalance between the two sites of the cluster in the presence of the potentials of the remaining electrons screened by the lattice polarization.

\section{B. Adiabatic hopping}

In order to determine the polaron hopping rate, we now calculate the evolution of the dynamical variables $\sigma_{z}$ and $Q$ within the cluster in the presence of the local field $\xi$, which by assumption is fixed during the time of the hopping process. The electronic variable $\sigma_{z}$ evolves quantum mechanically through Eq. (30), while the phonon collective variable $Q$ is taken to evolve through the classical Ehrenfest Eq. (29)..$^{20}$ As a further approximation, the electron transition probability can be estimated by standard methods within the adiabatic formulation of Refs. 16, 17, and 21: if the electron dynamics is faster than the motion of the phonons, the quantum variable $\sigma_{z}$ is able to equilibrate at any given value of the classical $Q$ and can be integrated out. This defines the following effective potential:

$$
V_{\mathrm{ad}}(Q)=\frac{1}{2} k Q^{2}-\sqrt{(\xi / \sqrt{2}+g Q)^{2} / 2+t^{2}},
$$

which is valid at temperatures lower than the barrier $\Delta(\xi)$ defined below. In the polaronic regime, it has the double-well shape illustrated in Fig. 1(a). Within the adiabatic description, an electron at site 1 is associated to a phononic variable being at the equilibrium point $Q \simeq-g / \sqrt{2} k$. For the occurrence of a classical nondissipative motion to the neighboring well, the initial kinetic energy of $Q$ taken from a Maxwell distribution must exceed the relative maximum of $V_{\text {ad }}$. This defines an energy barrier

$$
\Delta(\xi)=\Delta_{P}+\frac{\xi}{2}+\frac{k \xi^{2}}{4 g^{2}}+\mathcal{O}\left(t^{2}\right),
$$

where $\Delta_{P}=g^{2} / 4 k-t$ is the activation barrier for independent adiabatic polarons. ${ }^{22}$ The rate of electron hops per unit time from site 1 to site 2 then acquires a characteristic thermally activated behavior,

$$
w(\xi)=\frac{\omega_{0}}{2 \pi} \exp \left[-\Delta(\xi) / k_{B} T\right] .
$$

We note that $\omega_{0}^{-1}$ is the time it takes for the classical coordinate to pass from the initial point at $Q \simeq-g / \sqrt{2} k$ to the final
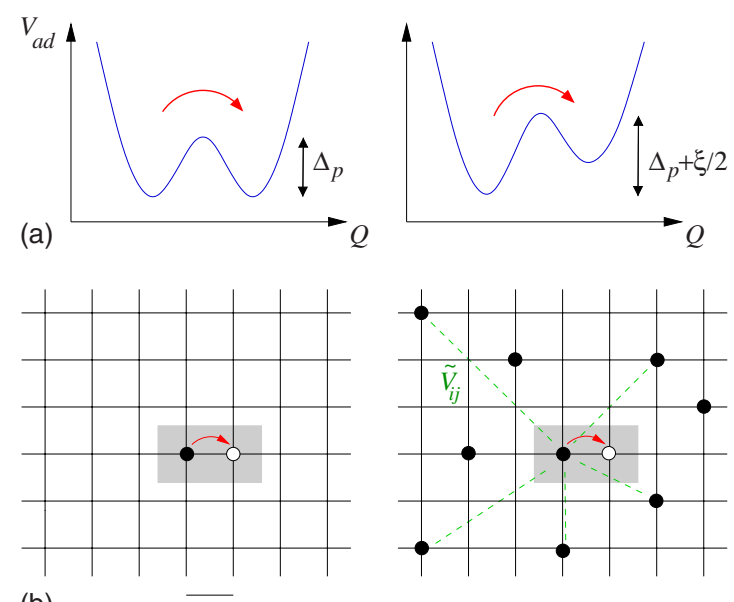

(b)

a

FIG. 1. (Color online) (a) Adiabatic double-well potential $V_{\text {ad }}(Q)$ of Eq. (34) for a two-site cluster, in the absence (left) and in the presence (right) of electron-electron interactions. The electron position follows the dynamics of the phonon coordinate $Q$ between the two minima of the potential. As pictorially represented in panel (b), interactions between the carriers modify the shape of the double well via the local field $\xi$, which measures the energy unbalance between the two sites due to the presence of the environment electrons. The grid represents the molecular lattice sites and the shaded area is the two-site cluster. The initial and final sites of the carrier hop are indicated, respectively, by a black dot and a white circle.

point at $Q \simeq g / \sqrt{2} k$ and therefore corresponds to the natural time scale of the hopping process. As can be seen from Eq. (36), in the hopping regime the time $w(\xi)^{-1}$ between hopping events is exponentially longer than the hopping timescale $\omega_{0}^{-1}$. This fact allows one to neglect the rearrangement of the environment electrons during a given hopping process, validating the cluster/environment separation scheme proposed in the present work.

Finally, we remark that the adiabatic treatment is valid when the condition

$$
\frac{\hbar \omega_{0}}{\pi}\left[\frac{\left(g^{2} / 4 k\right) k_{B} T}{\pi}\right]^{1 / 2} \ll t^{2}
$$

is met. ${ }^{16,17}$ In the opposite nonadiabatic regime, a hopping rate analogous to the one given by Eqs. (35) and (36) is obtained, with the following minor modifications: ${ }^{10,23-25}$ the prefactor $\left(\omega_{0} / 2 \pi\right)$ must be replaced by $p$ $=\left(t^{2} / \hbar^{2}\right)\left[\pi /\left(4 T \Delta_{P}\right)\right]^{1 / 2}$ and the barrier $\Delta_{P}=g^{2} / 4 k$. The nonadiabatic hopping rate has exactly the same dependence as Eq. (36) on the local field $\xi$ which embodies the effects of carrier-carrier interactions. The theory developed here for interacting small polarons therefore holds independently of the adiabatic/nonadiabatic character of the polaronic transport [Eq. (37)].

\section{Small-polaron mobility}

To determine the mobility, we assume that the current flow occurs through a succession of incoherent hopping events. Each individual process is characterized by a rate of the form Eq. (36), which depends explicilty on the electronic 
environment of the hopping particle through its own local field $\xi$. Neglecting the spatial fluctuations of the transition rate (36) amounts to averaging the hopping rate over all the possible values of the local field $\xi$ through the appropriate distribution $P(\xi)$. The mobility can then be written through Einstein's relation as

$$
\mu=\frac{e a^{2}}{k_{B} T}\langle w\rangle,
$$

where $a$ is the length of the electron hop, which we take to be equal to the intermolecular distance, and $\langle w\rangle$ is the statistical average, ${ }^{26}$

$$
\langle w\rangle=\int d \xi P(\xi) w(\xi) .
$$

To find the statistical distribution that enters in Eq. (39), we observe that if the system is sufficiently close to equilibrium, the value of the (static) local field $\xi$ is determined via Eq. (33) by the positions of the environment electrons prior to the hop. Correspondingly, $P(\xi)$ follows via Eq. (26) from the equilibrium distribution of interacting classical particles constrained to the presence of an electron on the initial cluster site. Such constraint clearly introduces spatial correlations between the hopping particle and the environment electrons. By creating a "correlation hole" around each carrier, interactions make polaron hopping in a finite density liquid more unfavorable than for noninteracting polarons, implying a reduction in the mobility. As will be shown in Sec. III D, such static correlations are reflected in an increase in the activation barrier for electrical transport. On the other hand, having implicitly assumed that the environment of any given particle is at equilibrium (i.e., that it relaxes to equilibrium before the same particle can hop again), we are automatically excluding dynamic correlations between subsequent hops. ${ }^{15}$ Preliminary numerical simulations performed by us on the interacting liquid indicate that such dynamic correlations can at most modify the prefactor of Eq. (38), which amounts to logarithmic corrections to the activation barrier. For the present problem of interacting polarons, the effect would therefore be negligible compared to the effect of spatial correlations that we are actually calculating.

Finally, the textbook result ${ }^{10}$ for the mobility of independent polarons is recovered by letting $\xi=0$ in the above equations,

$$
\mu_{P}=p \frac{e a^{2}}{k_{B} T} e^{-\Delta_{P} / k_{B} T} .
$$

\section{Mean-field approximation}

A complete determination of the statistical distribution $P(\xi)$ defined in Secs. II A and III C requires the knowledge of all the many-particle correlation functions of the system (generally speaking, the $n$th moment of the distribution is related to an $n$-particle correlation function). To obtain a tractable expression for the mobility, here we evaluate the effect of electron-electron interactions on average hopping rate (39) at mean-field level, i.e., neglecting the fluctuations of the local field $\xi$. This scheme of approximation corresponds to the theory applied in Ref. 9 to the study of organic/dielectric interfaces. It amounts to substituting averaged hopping rate (39) with its first cumulant,

$$
\langle w\rangle \simeq p \exp \left[-\Delta(\langle\xi\rangle) / k_{B} T\right] .
$$

With this replacement, the problem can be solved in terms of the sole two-particle correlation function of the interacting system through the evaluation of the average local field

$$
\langle\xi\rangle=\sum_{j}^{(\bar{c})}\left[\tilde{V}_{2, j}-\tilde{V}_{1, j}\right]\left\langle n_{j}\right\rangle_{1},
$$

where the symbol $\left\langle n_{j}\right\rangle_{1}$ stands for the constrained probability of occupation of site $j$ with site 1 occupied. As anticipated earlier, while the unconstrained average of $\xi$ would clearly vanish by symmetry in a homogeneous system, the spatial correlations enforced by this constraint cause a net additional energy $\operatorname{cost}\langle\xi\rangle>0$ for hopping from site to site in the presence of repulsive interactions. Assuming that the interaction correction $\langle\xi\rangle \lesssim \Delta_{P}$, so that the quadratic term $\xi^{2}$ in Eq. (35) can be neglected, we obtain a barrier

$$
\Delta(\langle\xi\rangle)=\Delta_{P}+\frac{\langle\xi\rangle}{2},
$$

which is the sum of the polaronic activation energy and a many-body correction term due to interactions. From Eq. (41) the density-dependent mobility can finally be expressed in terms of the mobility of independent polarons Eq. (40) as

$$
\mu=\mu_{P} \exp \left[-\langle\xi\rangle / 2 k_{B} T\right] .
$$

This result shows that in the regime $\langle\xi\rangle \lesssim \Delta_{P}$, the many-body effects on the mobility are completely decoupled from the individual polaron properties.

It can be noted that Eqs. (43) and (44) are formally equivalent to the formulas commonly used to describe impurity conduction in compensated polar semiconductors and in transition-metal oxide glasses. ${ }^{10,23,27,28}$ In such disordered systems, however, the microscopic mechanism responsible for the increase in the polaronic barrier is extrinsic to the polaronic system, as it originates from the ability of the particles to find an efficient percolating path connecting dilute and randomly distributed impurities. ${ }^{29}$ That picture is fundamentally different from the one considered here, where $\langle\xi\rangle$ originates from the mutual interactions between carriers in a perfectly crystalline material.

\section{LONG-RANGE COULOMB INTERACTIONS}

We now apply the theory developed so far to the calculation of the mobility of a liquid of small polarons in the presence of Coulomb interactions. We shall treat separately the cases of interacting polarons in two and three space dimensions. The former applies to the problem of polar interfaces as can be found in OFETs with highly polarizable gate dielectrics, while the latter can be relevant for doped polar semiconductors and oxides with strong electron-phonon interactions. In both situations, the hopping motion associated 
to the polaronic nature of the charge carriers prevents a proper screening of the interactions, so that the full longranged Coulomb potential needs to be considered. We shall therefore take the general form

$$
\tilde{V}_{i j}=\frac{\left(e^{*}\right)^{2}}{R_{i j}},
$$

where the effective charge $e^{*}$ accounts for the dielectric screening of the polar medium. It is shown in Appendix B that $e^{*}=e \sqrt{2 /\left(\kappa+\epsilon_{s}\right)}$ at a two-dimensional (2D) polar interface and $e^{*}=e / \sqrt{\epsilon_{s}}$ in a bulk polar material.

We start from the observation that for a Coulomb system, the correlation function $\left\langle n_{j}\right\rangle_{1}$ appearing in Eq. (42) varies on length scales set by the average interparticle distance $\sim R_{S}$ defined as $R_{s}=(\pi n)^{-1 / 2}$ in two dimensions and $R_{s}$ $=(4 \pi n / 3)^{-1 / 3}$ in three dimensions, $n$ being the particle density. At sufficiently low concentrations, $R_{s}$ is much larger than the lattice spacing so that this function can be safely replaced by its continuous limit. Correspondingly, the discrete sum appearing in Eq. (42) can be replaced by the following integral:

$$
\langle\xi\rangle=n \int d \mathbf{r}\left[\tilde{V}\left(\mathbf{r}+\mathbf{R}_{12}\right)-\tilde{V}(\mathbf{r})\right] g^{(2)}(r),
$$

with $R_{12}=a$ and $g^{(2)}(r)$ as the pair distribution function ${ }^{30}$ of a classical liquid of interacting charged particles-the onecomponent plasma (OCP). The properties of the OCP are governed by a single dimensionless coupling parameter,

$$
\Gamma=\frac{\left(e^{*}\right)^{2} / R_{s}}{k_{B} T},
$$

measuring the ratio between the electrostatic interactions and the thermal energy. This parameter identifies a weakly correlated and a strongly correlated regime, respectively, for $\Gamma$ $\ll 1$ and $\Gamma \gg 1$. Upon expanding the term between brackets in Eq. (46) to second order in $y=a / R_{s}$, it is readily shown that $\langle\xi\rangle$ can be expressed in terms of the dimensionless quantities $\Gamma$ and $y$ as

$$
\langle\xi\rangle=\frac{k_{B} T}{2} y^{2} F(\Gamma),
$$

with $F(\Gamma)$ as a universal function of the OCP. The manybody effects on the activation barrier are therefore entirely controlled by the parameter $\Gamma$ characterizing the interacting liquid.

\section{A. 2D}

For a homogeneous two-dimensional system, performing the angular integration in Eq. (46) and integrating the resulting expression by parts, we obtain

$$
F(\Gamma)=\Gamma \int_{0}^{\infty} d y \frac{g^{(2)}(y)}{y^{2}} .
$$

In the low-density/weakly interacting regime $\Gamma \ll 1$, the correlations of the classical OCP are fully determined by the Debye-Hückel form ${ }^{31}$

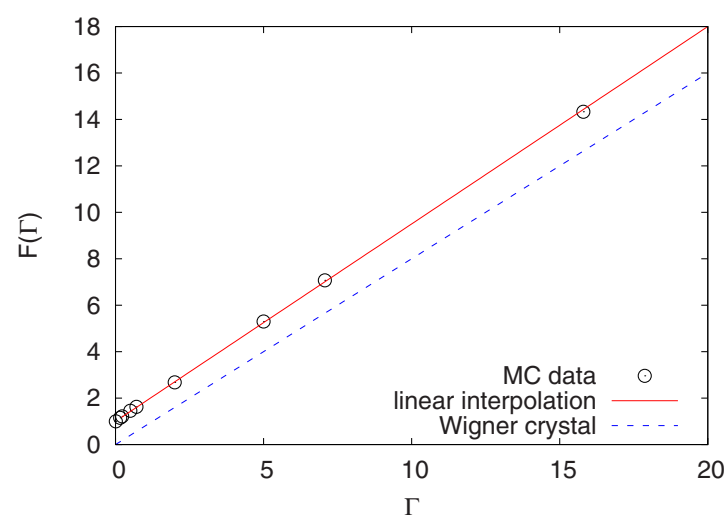

FIG. 2. (Color online) The function $F(\Gamma)$ for a classical twodimensional Coulomb liquid: Monte Carlo data of Ref. 33 (open circles), interpolating function (52) (full line), and Wigner crystal result (dashed line).

$$
g^{(2)}(r)=e^{-\tilde{V}(r) / k_{B} T}=e^{-\Gamma / y} .
$$

Upon substituting this function into Eq. (49), one obtains $F(\Gamma)=1$.

In the opposite limit of strong coupling, the electronic system undergoes Wigner crystallization, which occurs for $\Gamma>125 .{ }^{31}$ In this regime, it is easy to calculate the energy corresponding to a spatial displacement $u$ of a given electron while the remaining particles are kept at rest. Since the electron under study is initially in an equilibrium position, the energy variation is quadratic in the displacement and can be written as

$$
E(u)-E(0)=\zeta \frac{\left(e^{*}\right)^{2}}{2 R_{s}^{3}} u^{2} .
$$

Substituting $u=a$ and converting into the proper units, we obtain $F(\Gamma)=\zeta \Gamma$. The value of the numerical constant $\zeta=0.8$ has been obtained through direct Ewald summation of the Coulomb interactions on a triangular lattice, ${ }^{32}$ which is the lowest energy structure of a Wigner crystal in two dimensions.

For the evaluation of $\langle\xi\rangle$ at intermediate interaction strengths, we resort to the Monte Carlo simulations of the classical two-dimensional OCP performed in Ref. 33. There the pair distribution function $g^{(2)}(r)$ was tabulated at different values of the Coulomb interaction parameter. Upon performing integral (49) using such numerical data, one obtains a discrete set of points for the function $F(\Gamma)$. In the range 1 $<\Gamma<20$, the result can be parametrized through the linear interpolating function,

$$
F(\Gamma)=1+0.85 \Gamma
$$

within $1 \%$ accuracy (cf. Fig. 2), and this formula remains fairly accurate even at larger values of $\Gamma$ until it eventually merges into the strong-coupling Wigner crystal estimate. It can be observed that except for a constant preasymptotic term of order 1 , the function $F(\Gamma)$ representing the interparticle correlations in the Wigner crystal has essentially the same $\Gamma$ dependence as that of the correlated liquid. Using Eqs. (47) and (52) and the definition of $R_{s}$, we can finally 


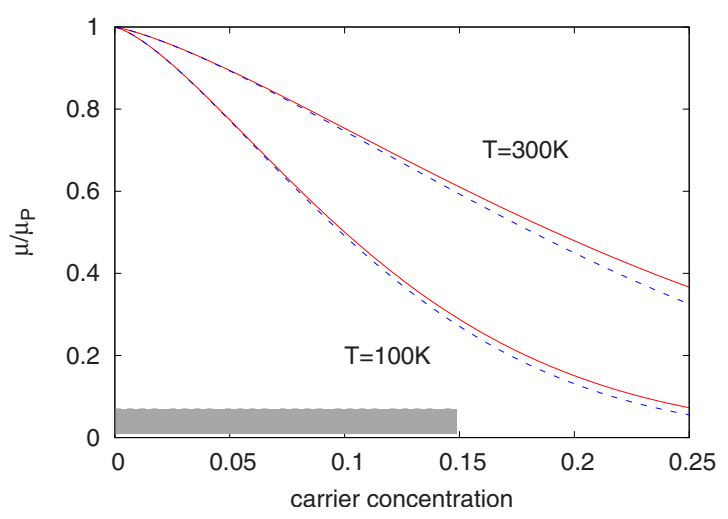

FIG. 3. (Color online) Interaction-induced reduction in the polaronic mobility calculated with the parameters appropriate to a rubrene $/ \mathrm{Ta}_{2} \mathrm{O}_{5}$ interface at two different temperatures. The shaded area represents the range of densities studied in Ref. 9. The full curves are obtained from Eq. (44), while the dashed curves include the full activation barrier given by Eq. (35) with $\Delta_{P}=55 \mathrm{meV}$. The differences are negligible because in all the explored range $\langle\xi\rangle$ $\lesssim \Delta_{P}$. Similar curves are obtained for bulk transition-metal oxides.

write the many-body correction to the polaronic activation barrier due to Coulomb interactions as

$$
\langle\xi\rangle=\frac{\pi}{2} n a^{2}\left[k_{B} T+0.85\left(e^{*}\right)^{2}(\pi n)^{1 / 2}\right] .
$$

The average local field becomes temperature independent and behaves asymptotically as $\langle\xi\rangle \propto n^{3 / 2}$ in the strongly correlated limit $(\Gamma \gg 1)$, i.e., when the second term between brackets dominates.

It was shown in Refs. 2 and 9 that carriers in rubrene $/ \mathrm{Ta}_{2} \mathrm{O}_{5}$ devices form small polarons. Considering the effective polaron-polaron interaction derived in Appendix B for organic/dielectric interfaces and using the parameters appropriate to a rubrene $/ \mathrm{Ta}_{2} \mathrm{O}_{5}$ devices of Ref. $9(a=7.2 \AA$, $\epsilon_{s}=25$, and $\kappa=3$ ), we infer that a moderately correlated polaron liquid is realized in the two-dimensional conducting channel, with coupling parameters in the range $0<\Gamma \lesssim 9$. In this regime polaron-polaron correlations yield an increase in the activation barrier for transport reaching $\langle\xi\rangle / 2$ $\sim 13 \mathrm{meV}$ at the highest concentrations measured $(x$ $\sim 0.15$ ). This is smaller than the barrier $\Delta_{P}=55 \mathrm{meV}$ for independent polarons consistent with the assumptions underlying our derivation. When substituted into Eq. (44), such many-body correction leads to a sizable reduction in the polaronic mobility, as illustrated in Fig. 3 at two different temperatures. ${ }^{34}$

\section{B. 3D}

In three space dimensions,

$$
F(\Gamma)=\Gamma \int_{0}^{\infty} d y \frac{d}{d y} g^{(2)}(y)=\Gamma .
$$

This result, which follows directly from the fact that in a homogeneous liquid phase $g^{(2)}(\infty)=1$ (the pair correlations vanish at large distances), holds exactly at all $\Gamma$. It is there- fore not necessary to integrate numerically the pair distribution function obtained from Monte Carlo simulations as was done in the two-dimensional case. It can be directly checked that result (54) also extends to the crystallized phase. To this aim we observe that the energy cost to displace a particle from its equilibrium position in a three-dimensional Wigner crystal is still given by Eq. (51), with now $\zeta^{(3 \mathrm{D})}=1$ from Gauss's theorem, ${ }^{35}$ also leading to $F(\Gamma)=\Gamma$.

For practical calculations the result can be rewritten in terms of the carrier density $n$ in a generic three-dimensional system as

$$
\langle\xi\rangle=\frac{2 \pi}{3} \frac{e^{2}}{\epsilon_{s}} n a^{2},
$$

which is obtained by substituting Eq. (54) into Eq. (48). As usual, $a$ is the hopping distance, on the order of the lattice spacing. The linear density dependence of the local field resulting from Eq. (55) is weaker than the $n^{3 / 2}$ behavior obtained in two dimensions and is temperature independent at all densities.

Using typical values for transition-metal oxides, such as $a=4 \AA$ and $\epsilon_{s}=10-100$ and assuming a cubic lattice structure for simplicity, we obtain a barrier increase $\langle\xi\rangle / 2=\alpha x$, where $x$ is the carrier concentration and the coefficient $\alpha$ $\simeq 40-400 \mathrm{meV}$. An increase in the activation energy with electron concentration compatible with such prediction has been observed in doped three-dimensional transition-metal oxides exhibiting small-polaron conduction such as magnetite $^{36}$ and the manganites. ${ }^{37,38}$ Actually, in the hightemperature phases of the manganite compounds $\mathrm{La}_{x} \mathrm{Ca}_{1-x} \mathrm{MnO}_{3}$, both a large-polaron scenario (in bulk samples ${ }^{39}$ ) and a small-polaron scenario (in thin films ${ }^{37}$ ) have have been invoked to interpret the transport properties in the lightly electron-doped regime. We have performed a linear fit of the doping dependence of the activation energy $\Delta$ reported in Ref. 37 in the range $0<x<0.35$, yielding $\Delta$ $=46+56 x \mathrm{meV}$. When compared with Eqs. (43) and (55), the fitted slope of the concentration-dependent term yields $\epsilon_{s} \simeq 70$, in good agreement with the dielectric constants measured in those compounds $\left(\epsilon_{s} \simeq 55-90\right.$ from Ref. 40).

\section{DISCUSSION AND CONCLUSIONS}

In this work we have derived a theory for the hopping transport of mutually interacting polarons in narrow-band materials. Observing that in the hopping regime the quantum coherence of the carriers extends over only few lattice sites, we solve for the quantum dynamics of the carriers within a finite-size cluster, taking into account the interactions with the other charges in the environment via a set of static fields. The calculation then proceeds by assuming that transport occurs through statistically independent hopping events. Correspondingly, the many-particle mobility is obtained from a statistical average of the intermolecular hopping rates over the distribution of environment fields, which follows from the known statistical properties of the interacting liquid.

The proposed decoupling scheme, which is analogous to the one followed by Pardee and Mahan ${ }^{14,15}$ in the context of ionic conductors, is justified here by the quasistatic nature of 
TABLE I. Summary of the main formulas determining the density-dependent mobility of small polarons interacting through the long-range Coulomb potential of Eq. (45) in two and three dimensions. $R_{S}$ is the mean interparticle separation, $a$ is the hopping distance equal to the distance between molecular units, and $e^{*}$ is the effective charge determined by the dielectric environment (see Appendix B). The right column follows from Eqs. (47)-(49) and (54). It gives the mean-field correction to the polaron mobility due to many-body effects through $\mu / \mu_{P}=\exp \left(-\langle\xi\rangle / 2 k_{B} T\right)$ [Eq. (44)].

\begin{tabular}{ccc}
\hline \hline $2 \mathrm{D}$ & $R_{s}=(\pi n)^{-1 / 2}$ & $\langle\xi\rangle=\pi / 2 n a^{2}\left[k_{B} T+0.85\left(e^{*}\right)^{2}(\pi n)^{1 / 2}\right]$ \\
$3 \mathrm{D}$ & $R_{s}=(4 \pi n / 3)^{-1 / 3}$ & $\langle\xi\rangle=2 \pi / 3\left(e^{*}\right)^{2} n a^{2}$ \\
\hline
\end{tabular}

the carriers in the hopping regime as a consequence of polaronic self-trapping. Despite this simplification, which amounts to neglecting dynamical correlations between subsequent hops, the spatial correlations between particles which constitute the dominant many-body effects on polaronic transport are fully retained. When applied to a liquid of small polarons interacting through long-range Coulomb forces, the theory predicts a net increase in the activation barrier for electrical transport and hence a reduction in the carrier mobility. The analytical formulas obtained at meanfield level, i.e., neglecting the fluctuations of the environment field $\xi$ representing the polaron-polaron correlations, are summarized in Table I.

The present scenario consistently explains the current characteristics of rubrene/ $\mathrm{Ta}_{2} \mathrm{O}_{5}$ OFETs measured in Ref. 9. There, a saturation of the usual linear $I \propto V_{g}$ relationship expected for independent carriers was observed at large values of the gate voltage $V_{g}$, indicative of a sizable reduction in the mobility (the reader is referred to that work for a detailed comparison with the experimental data). An interpretation in terms of carrier-carrier interactions comes naturally in these devices where, as was mentioned in Sec. I, all the conditions for the observation of the predicted many-body effects on the polaronic hopping transport are simultaneously met: smallpolaron formation (because of the strong polar coupling with the gate dielectric and the narrow bandwidth of the organic semiconductor), long-range Coulomb repulsion between the carriers, and broad tunability of the carrier concentration via the applied gate potential.

We anticipate based on our theoretical results that, in principle, nothing prevents the observation of a downturn of the $I-V_{g}$ curves beyond the saturation regime observed in Ref. 9 . For this, the only requirement is that of a stronger reduction in the mobility than the one realized at rubrene $/ \mathrm{Ta}_{2} \mathrm{O}_{5}$ interfaces. As is clear from Fig. 3, this can be achieved either by reducing the temperature or by increasing the carrier density, as both effects lead to an increase in the correlation parameter $\Gamma$ [see Eq. (47)] and therefore of the ratio $\langle\xi\rangle / 2 k_{B} T$ in Eq. (44). An interesting possibility in this direction is offered by the use of polar electrolytes as gate materials, allowing to reach much higher concentrations than with conventional polar dielectrics. ${ }^{41,42}$

Finally, due to the very general nature of the mechanisms involved, one might ask if similar effects can be observed in other classes of systems. In principle, any system with a sufficient concentration of small polarons (whatever the mi- croscopic origin) interacting through long-range repulsive forces should exhibit a density-dependent increase in the transport activation energy. In fact, we have found at least two examples in the literature which could fit in the present scenario. In the manganite compound $\mathrm{La}_{x} \mathrm{Ca}_{1-x} \mathrm{MnO}_{3}$, systematic experimental studies of polaronic transport in both thin films ${ }^{37}$ and bulk samples ${ }^{38}$ have reported a monotonic increase in the activation barrier upon increasing the electron concentration $x$ that could be ascribed to polaron-polaron interactions. ${ }^{38}$ An analogous increase/decrease in activation energy has been observed upon increasing/decreasing the electron concentration in magnetite via $\mathrm{Ti}$ and $\mathrm{Zn}$ dopings, respectively. ${ }^{36}$ A possible explanation in terms of long-range Coulomb interactions between the carriers has also been explicitly suggested. In both classes of compounds, the linear dependence of the activation energy with electron doping is indeed compatible with the predictions of our theory. Nevertheless, other mechanisms cannot be excluded-related to the complex structural details of these materials, to the effectiveness of electronic screening, as well as to the presence of randomly distributed ionized dopants, whose electric fields could also affect the polaronic hopping rates. These mechanisms could be responsible for the more complex phenomenology observed in other systems, as, for example, in boron carbides, ${ }^{43}$ where both a doping-independent activation as well as an activation barrier decreasing with concentration have been reported depending on the explored temperature range.

We conclude by suggesting an experimental method that could be useful to disentangle more clearly the effects of polaron-polaron interactions from the intrinsic features of noninteracting polarons. Such method relies on the comparison of the activation energy $\Delta$ determined from electrical transport and $\Delta_{S}$ obtained from thermoelectric power measurements. Since the thermopower is insensitive to a uniform polaronic renormalization of the carriers, ${ }^{44} \Delta_{S}$ would give a direct measure of the interaction correction $\langle\xi\rangle / 2$ alone, while electrical transport would be governed by the sum $\Delta$ $=\Delta_{P}+\langle\xi\rangle / 2$. Such method has been often applied to disentangle impurity effects from polaron effects in transitionmetal oxide glasses ${ }^{28,45}$ and has also been proposed in the context of ionic conductors. ${ }^{15,46}$ Comparative analysis of the electrical and thermal transport has also been performed in the manganite compounds to ascertain the polaronic nature of the charge carriers. ${ }^{38,47}$ The feasibility of thermoelectric power measurements in OFETs has been recently demonstrated in Ref. 48 and could provide further independent insight into the many-body physics of organic field-effect transistors. An extension of the present theory to include the effects of polaron-polaron correlations beyond the mean-field approximation, as well as its generalization to disordered systems, is underway.

\section{ACKNOWLEDGMENTS}

The authors acknowledge useful discussions with M. J. Calderón, A. F. Morpurgo, C. Pierleoni, and A. Troisi. S.F. received financial support from CONSOLIDER under Grant No. CSD2007-0010, and S.C. from the Research Program MIUR-PRIN 2005. 


\section{APPENDIX A: HAMILTONIAN OF A TWO-SITE CLUSTER}

For a two-site cluster, Hamiltonian (24) explicitly reads

$$
\begin{aligned}
H_{\mathrm{cl}}= & H_{t}+V_{1,2} n_{1} n_{2}+Y_{1} \eta_{1}+Y_{2} \eta_{2}+n_{1}\left(\epsilon_{1}+Y_{1}\right)+n_{2}\left(\epsilon_{2}+Y_{2}\right) \\
& +\frac{k}{2\left(\left[g^{2}\right]_{11}^{2}-\left[g^{2}\right]_{12}^{2}\right)}\left\{\left[g^{2}\right]_{11}\left(Y_{1}^{2}+Y_{2}^{2}\right)-2\left[g^{2}\right]_{12} Y_{1} Y_{2}\right\} .
\end{aligned}
$$

For the present problem, we can assume without loss of generality that the cluster is singly occupied (there is one electron on the initial site, the other site being empty for the hopping process to be allowed). It is then possible to rewrite the cluster Hamiltonian in a form which is formally equivalent to that of a tunneling charge interacting with $a$ single effective mode, which is essentially a spin-boson model. Defining the couplings

$$
\begin{aligned}
& \bar{g}^{2}=\left[g^{2}\right]_{11}+\left[g^{2}\right]_{12}, \\
& g^{2}=\left[g^{2}\right]_{11}-\left[g^{2}\right]_{12},
\end{aligned}
$$

introducing the variables

$$
\begin{aligned}
& x=\frac{Y_{2}-Y_{1}}{\sqrt{2 g}}, \\
& \epsilon=\frac{\epsilon_{2}-\epsilon_{1}}{\sqrt{2}}, \\
& \eta=\frac{\eta_{2}-\eta_{1}}{\sqrt{2}},
\end{aligned}
$$

and enforcing the single occupancy within the cluster through the condition $n_{1}+n_{2}=1$, we can rewrite Eq. (A1) as

$$
H_{\mathrm{cl}}=H_{t}-\epsilon \frac{n_{1}-n_{2}}{\sqrt{2}}-g x\left(\frac{n_{1}-n_{2}}{\sqrt{2}}-\eta\right) \frac{1}{2} k x^{2}+E^{\prime},
$$

where, for single occupancy, $E^{\prime}$ is a constant. From Eq. (A7) we see that the variable $\eta$ only contributes to an unimportant shift in the $x$ equilibrium position. It is therefore convenient to introduce the deviation $Q=x-g \eta / k$ as well as a different interaction variable,

$$
\xi=\sqrt{2}\left(\epsilon-g^{2} \eta / k\right),
$$

which takes into account both the elecrton-electron interaction and the electron-phonon screening correction.

Dropping all terms which do not couple to the site occupations or to the phonon displacement and introducing the pseudospin notation $\sigma_{z}=n_{1}-n_{2}$ and $H_{t}=-t \sigma_{x}$, we arrive at Eq. (30).

\section{APPENDIX B: EFFECTIVE ELECTRON-ELECTRON INTERACTIONS}

\section{Organic/dielectric interfaces}

In organic field-effect transistors, charge carriers accumulate in a two-dimensional layer located at the interface be- tween an organic crystal and a polar gate dielectric. ${ }^{1}$ Model (1) therefore consists of two-dimensional tight-binding electrons interacting with the polar-phonon modes of the interface. In Fourier space, the electron-phonon interaction matrix element has the simple form ${ }^{3-5,49}$

$$
M_{q}=M_{0} e^{-q z} / \sqrt{q},
$$

where $q$ is the momentum parallel to the interface, $z$ is the distance of the electrons to the polar interface, which acts as a short-distance cutoff, and $M_{0}$ is a coupling constant that depends on the dielectric properties of the interface. ${ }^{50}$ It is given by $M_{0}^{2}=2 \pi \hbar \omega_{0} e^{2} \beta / S$, with $S$ as the total surface of the system, and $\omega_{0}$ as the frequency of the coupled dispersionless polar mode. The parameter $\beta$ is a combination of the known dielectric constants of the two media that constitute the interface, which determines the strength of the electronphonon coupling. In the present example of an organic/ dielectric interface, $\beta=\left(\epsilon_{s}-\epsilon_{\infty}\right) /\left(\epsilon_{s}+\kappa\right) /\left(\epsilon_{\infty}+\kappa\right)$, where $\kappa$ is the (frequency independent) dielectric constant of the organic semiconductor, and $\epsilon_{s}, \epsilon_{\infty}$ are, respectively, the static and high-frequency dielectric constants of the polarizable dielectric.

We start with the "bare" interaction potential $V_{i j}$ between two charges located at a distance $z$ from the interface:

$$
V_{i j}=\frac{e^{2}}{\kappa}\left[\frac{1}{R_{i j}}-\frac{1}{\sqrt{R_{i j}^{2}+4 z^{2}}} \frac{\epsilon_{\infty}-\kappa}{\epsilon_{\infty}+\kappa}\right],
$$

where $\epsilon_{\infty}$ accounts for the high-frequency electronic polarizability of the polar material. To determine the effective potential, we evaluate

$$
\frac{\left[g^{2}\right]_{i j}}{k}=\frac{\int \frac{d^{2} q}{(2 \pi)^{2}} e^{-i q R_{i j} M_{q}^{2}}}{k=2 \beta e^{2} \frac{1}{\sqrt{R_{i j}^{2}+4 z^{2}}}}
$$

and with Eq. (21), we obtain

$$
\tilde{V}_{i j}=\frac{e^{2}}{\kappa}\left[\frac{1}{R_{i j}}-\frac{1}{\sqrt{R_{i j}^{2}+4 z^{2}}} \frac{\epsilon_{s}-\kappa}{\epsilon_{s}+\kappa}\right] .
$$

This result is equivalent to what one would obtain from a simple image charge calculation, considering the full static dielectric constant $\epsilon_{s}$ of the polar material right from the beginning. ${ }^{32}$

It was shown in Ref. 2 that the conduction in organic FETs effectively takes place within the first molecular layer nearby the interface. The cut-off distance $z$ is therefore on the order of the lateral size of the molecules, which is comparable with the lattice spacing $a$ itself. At concentrations such that the typical interparticle spacing $R_{s}$ is much larger than both $a$ and $z$, effective interaction potential (B4) reduces to

$$
\tilde{V}_{i j}=\frac{2}{\epsilon_{s}+\kappa} \frac{e^{2}}{R_{i j}} .
$$

which corresponds to a long-ranged Coulomb potential with a screened charge $e^{*}=e \sqrt{2 /\left(\epsilon_{s}+\kappa\right)}$. 


\section{Bulk polar materials}

In three-dimensional polar systems, one starts with the bare interaction potential

$$
V_{i j}=\frac{e^{2}}{\epsilon_{\infty} R_{i j}},
$$

where $\epsilon_{\infty}$ accounts for the high-frequency polarizability of the material. The interaction of the electrons with the polarphonon modes is described by the Fröhlich matrix element $M_{q}=M_{0} / q$, with $M_{0}^{2}=2 \pi \hbar \omega_{0}\left(e^{2} / \widetilde{\epsilon}\right) / \Omega$. Here $\Omega$ is the total volume of the system, $\omega_{0}$ is the frequency of the coupled dispersionless phonon mode, and $\tilde{\epsilon}=\left(\epsilon_{\infty}^{-1}-\epsilon_{s}^{-1}\right)^{-1}$ is an effective dielectric constant. Including the screening effect of the polar modes as given by Eq. (16) correctly yields

$$
\tilde{V}_{i j}=\frac{e^{2}}{\epsilon_{s} R_{i j}},
$$

corresponding to a screened charge $e^{*}=e / \sqrt{\epsilon_{s}}$.

\section{Local interactions}

To conclude this appendix, we observe that local electronphonon interactions as the ones described by the Holstein model do not give rise to a long-range screening term. This can be readily seen from Eq. (21), where $\left[g^{2}\right]_{i j} / k \propto \delta_{i j}$. The effective electron-electron interactions are therefore of the unscreened form $V_{i j}=e^{2} / \epsilon_{\infty} R_{i j}$ in bulk materials and $V_{i j}$ $=2 e^{2} /\left(\kappa+\epsilon_{\infty}\right) R_{i j}$ at interfaces. For a given carrier density, the coupling parameter $\Gamma$ is therefore larger than in the case of polar screening, and the interaction effects on the mobility should be correspondingly enhanced.
${ }^{1}$ M. E. Gershenson, V. Podzorov, and A. F. Morpurgo, Rev. Mod. Phys. 78, 973 (2006).

${ }^{2}$ I. N. Hulea, S. Fratini, H. Xie, C. L. Mulder, N. N. Iossad, G. Rastelli, S. Ciuchi, and A. F. Morpurgo, Nature Mater. 5, 982 (2006).

${ }^{3}$ S. Q. Wang and G. D. Mahan, Phys. Rev. B 6, 4517 (1972).

${ }^{4}$ K. Hess and P. Vogl, Solid State Commun. 30, 807 (1979).

${ }^{5}$ N. Mori and T. Ando, Phys. Rev. B 40, 6175 (1989).

${ }^{6}$ M. V. Fischetti, D. A. Neumayer, and E. A. Cartier, J. Appl. Phys. 90, 4587 (2001)

${ }^{7}$ R. Chau, S. Datta, M. Doczy, B. Doyle, J. Kavalieros, and M. Metz, IEEE Electron Device Lett. 25, 408 (2004).

${ }^{8}$ S. Fratini and F. Guinea, Phys. Rev. B 77, 195415 (2008).

${ }^{9}$ S. Fratini, H. Xie, I. N. Hulea, S. Ciuchi, and A. F. Morpurgo, New J. Phys. 10, 033031 (2008).

${ }^{10}$ I. G. Austin and N. F. Mott, Adv. Phys. 18, 41 (1969).

${ }^{11}$ A. Ohtomo and H. Y. Hwang, Nature (London) 427, 423 (2004).

${ }^{12}$ H. Alves, A. S. Molinari, H. Xie, and A. F. Morpurgo, Nature Mater. 7, 574 (2008).

${ }^{13}$ C. H. Ahn, A. Bhattacharya, M. Di Ventra, J. N. Eckstein, C. Daniel Frisbie, M. E. Gershenson, A. M. Goldman, I. H. Inoue, J. Mannhart, A. J. Millis, A. F. Morpurgo, D. Natelson, and J.-M. Triscone, Rev. Mod. Phys. 78, 1185 (2006).

${ }^{14}$ W. J. Pardee and G. D. Mahan, J. Solid State Chem. 15, 310 (1975).

${ }^{15}$ G. D. Mahan, Phys. Rev. B 14, 780 (1976).

${ }^{16}$ T. Holstein, Ann. Phys. (N.Y.) 8, 343 (1959).

${ }^{17}$ I. G. Lang and Yu. A. Firsov, Sov. Phys. Solid State 9, 2701 (1968).

${ }^{18}$ F. Bassani, M. Geddo, G. Iadonisi, and D. Ninno, Phys. Rev. B 43, 5296 (1991).

${ }^{19}$ G. Verbist, M. A. Smondyrev, F. M. Peeters, and J. T. Devreese, Phys. Rev. B 45, 5262 (1992).

${ }^{20}$ S. Paganelli and S. Ciuchi, J. Phys.: Condens. Matter 20, 235203 (2008).

${ }^{21}$ D. Emin and T. Holstein, Ann. Phys. (N.Y.) 53, 439 (1969).

${ }^{22}$ The barrier can be expressed in terms of the polaron energy as $g^{2} / 4 k=\gamma E_{P}$, with $\gamma=\left(1-\left[g^{2}\right]_{12} /\left[g^{2}\right]_{11}\right) / 2 \leq 1 / 2$, the equality holding for purely local electron-phonon interactions (Ref. 35).
For the Fröhlich interaction in three dimensions, a straightforward calculation using the Fourier transform of the matrix element $M_{q} \propto 1 / q$ on a cubic lattice gives $\gamma \simeq 0.3$, while for the electron-phonon interaction at polar interfaces, $\quad \gamma \simeq 1 / 2$ $-z / \sqrt{a^{2}+4 z^{2}}$ monotonically decreases with the distance $z$ to the interface.

${ }^{23}$ J. Schnakenberg, Phys. Status Solidi 28, 623 (1968).

${ }^{24}$ R. A. Marcus, Rev. Mod. Phys. 65, 599 (1993).

${ }^{25}$ V. Coropceanu, J. Cornil, D. A. da Silva Filho, Y. Olivier, R. Silbey, and J.-L. Brédas, Chem. Rev. 107, 926 (2007).

${ }^{26}$ Equation (39) has the meaning of a spatial average over the fields $\xi$ encountered by the different particles that hop. These are treated as static variables in virtue of the decoupling of time scales discussed after Eq. (36). Relaxing this approximation could modify qualitatively the rate process beyond Eq. (39) [see R. Zwanzig, Acc. Chem. Res. 23, 148 (1990)].

${ }^{27}$ D. Emin, Phys. Rev. B 46, 9419 (1992).

${ }^{28}$ L. Murawski, C. H. Chung, and J. D. Mackenzie, J. Non-Cryst. Solids 32, 91 (1979).

${ }^{29}$ A. Miller and E. Abrahams, Phys. Rev. 120, 745 (1960).

${ }^{30}$ J. P. Hansen and I. R. McDonald, Theory of Simple Liquids (Academic, London, 1976).

${ }^{31}$ S. Ichimaru, Rev. Mod. Phys. 54, 1017 (1982).

${ }^{32}$ S. Fratini, A. F. Morpurgo, and S. Ciuchi, J. Phys. Chem. Solids 69, 2195 (2008).

${ }^{33}$ H. Totsuji, Phys. Rev. A 17, 399 (1978).

${ }^{34}$ The condition for adiabatic transport is fulfilled in such devices, as the ratio between the left-hand side and the right-hand side of Eq. (37) is $\sim 0.3$ at room temperature. Actually such value places these devices close to the adiabatic/nonadiabatic crossover, where the polaronic activation energy changes from $\Delta_{P}$ $=\left(g^{2} / 4 k\right)-t$ to the nonadiabatic value $\Delta_{P}=\left(g^{2} / 4 k\right)$. This can be at the origin of the missing correction $-t$ in the activation energy reported in Refs. 2 and 9.

${ }^{35}$ G. D. Mahan, Many-Particle Physics, 3rd ed. (Plenum, New York, 2000).

${ }^{36}$ A. Kozłowski, R. J. Rasmussen, J. E. Sabol, P. Metcalf, and J. M. Honig, Phys. Rev. B 48, 2057 (1993).

${ }^{37}$ D. C. Worledge, L. Miéville, and T. H. Geballe, Phys. Rev. B 57, 
15267 (1998).

${ }^{38}$ T. T. M. Palstra, A. P. Ramirez, S. W. Cheong, B. R. Zegarski, P. Schiffer, and J. Zaanen, Phys. Rev. B 56, 5104 (1997).

${ }^{39}$ J. L. Cohn, C. Chiorescu, and J. J. Neumeier, Phys. Rev. B 72, 024422 (2005).

${ }^{40}$ J. L. Cohn, M. Peterca, and J. J. Neumeier, Phys. Rev. B 70, 214433 (2004).

${ }^{41}$ H. Shimotani, H. Asanuma, J. Takeya, and Y. Iwasa, Appl. Phys. Lett. 89, 203501 (2006).

${ }^{42}$ M. J. Panzer and C. D. Frisbie, J. Am. Chem. Soc. 129, 6599 (2007).

${ }^{43}$ T. L. Aselage, D. Emin, and S. S. McCready, Phys. Rev. B 64, 054302 (2001).

${ }^{44}$ D. Emin, Phys. Rev. Lett. 35, 882 (1975).

${ }^{45}$ M. J. Burns and P. M. Chaikin, J. Phys. C 18, L743 (1985).
${ }^{46}$ S. M. Girvin, J. Solid State Chem. 25, 65 (1978).

${ }^{47}$ M. Jaime, M. B. Salamon, M. Rubinstein, R. E. Treece, J. S. Horwitz, and D. B. Chrisey, Phys. Rev. B 54, 11914 (1996).

${ }^{48}$ K. P. Pernstich, B. Rössner, and B. Batlogg, Nature Mater. 7, 321 (2008).

${ }^{49}$ J. Sak, Phys. Rev. B 6, 3981 (1972).

${ }^{50}$ Interaction (B1) was derived from the macroscopic laws of electrostatics that are valid at distances $>a$. In real interfaces, the discrete nature of the polarizable medium should lead to an additional short-range cutoff at lengths on the order of the interionic spacing. To a first approximation, this effect can be incorporated by treating $z$ as an effective phenomenological quantity which includes both the channel-interface distance and the lattice cutoff. 